\title{
MINIMAL DEGREES OF FAITHFUL CHARACTERS OF FINITE GROUPS WITH A T.I. SYLOW $p$-SUBGROUP
}

\author{
T. R. BERGER, P. LANDROCK, AND G. O. MICHLER
}

\begin{abstract}
Using the classification of the finite simple groups we show in this article that a faithful complex character $\chi$ of a finite group $G$ with a nonnormal T.I. Sylow $p$-subgroup $P$ has degree $\chi(1)>\sqrt{|P|}-1$. This result verifies a conjecture of $\mathrm{H}$. S. Leonard [10].
\end{abstract}

Introduction. Let $p$ be a fixed prime, and let $G$ be a finite group with a T.I. Sylow $p$-subgroup $P$. That is, two different conjugates of $P$ have only the identity element in common. In [10] H. S. Leonard conjectured that if $G$ has a faithful complex character $\chi$ with degree $\chi(1) \leqslant \sqrt{|P|}-1$, then $P$ is normal in $G$. Using the classification of the finite simple groups we prove Leonard's conjecture in this note (Theorem 3.2).

In $\S 1$ this theorem is first proved for $p$-solvable groups $G$ (Proposition 1.3). Then we determine the composition series of a minimal counterexample $G$ to Leonard's conjecture (Proposition 1.4). Since by Sibley's theorem [12] the main result of this article is known if $P$ is cyclic, we give in $\$ 2$ a complete list of all finite simple groups $G$ having a noncyclic T.I. Sylow $p$-subgroup for some prime $p$ (Proposition 2.3). Here for odd $p$ we use Gorenstein and Lyons' theorem [4] classifying all finite groups $G$ with $O_{p^{\prime}}(G)=1, p$-rank $m_{p}(G)>1$, and containing a strongly $p$-embedded subgroup. If $p=2$, then Proposition 2.3 is only a restatement of Suzuki's theorem [13]. After these preparations Leonard's conjecture is proved in §3. In Remark 3.3 we show that the bound of Theorem 3.2 cannot be replaced by $\frac{1}{2}(|P|-1)$, which is the bound of Sibley's theorem [12].

For notation and terminology we refer to the books by Feit [1], Gorenstein [2,3], Huppert [5], Huppert and Blackburn [6], and Landrock [9]. All character tables of finite simple groups used here are contained in the CAS-system [11] of J. Neubüser, H. Pahlings, and W. Plesken (TH. Aachen, Federal Republic of Germany).

1. Reduction to almost simple groups. In this section we determine the structure of a finite group $G$ of minimal order among the groups $H$ without a normal Sylow p-subgroup, but satisfying the hypothesis of Leonard's conjecture.

The following lemma due to Feit [1, p. 123] is our basic tool.

Received by the editors March 10, 1985 and, in revised form, August 15, 1985.

1980 Mathematics Subject Classification (1985 Revision). Primary 20C05, 20C10, 20C11, 20C15, $20 \mathrm{C} 20$. 20F26; Secondary 20D05, 20E32, 20D20. 
LEMMA 1.1. Let $S$ be a splitting field of characteristic zero for the finite group $G$ with a T.I. Sylow p-subgroup $P$. Let $\chi$ be a character of $S G$ such that $\chi(1)^{2} \leqslant|P|$. Let $H$ be a subgroup of $G$ containing $N_{G}(P)$. Then $(\chi, \chi)_{G}=(\chi, \chi)_{H}$.

For a short proof of this result we refer to [9, p. 129].

Lemma 1.2. Let $G$ be a finite group with a T.I. Sylow p-subgroup P. Then the following assertions hold.

(a) Every subgroup $U$ of $G$ with $p|| U \mid$ has a T.I. Sylow p-subgroup.

(b) $G / N$ has a T.I. Sylow p-subgroup for every normal subgroup $N$ of $G$ with $(p,|N|)=1$.

(c) $C_{G}(x)$ has a normal Sylow p-subgroup for every $1 \neq x \in P$.

Proof. See Suzuki [13, p. 59].

Proposition 1.3. Let $G$ be a p-solvable group with T.I. Sylow p-subgroup $P$ and $a$ faithful complex character $\chi$ such that $\chi(1)^{2} \leqslant|P|$. Then $P$ is a normal subgroup of $G$.

Proof. Let $G$ be a minimal counterexample and let $(F=R / \pi, R, S=$ quot $(R))$ be a splitting $p$-modular system for $G$ (see [9, p. 47]). Then $O_{p}(G)=1$, and so $Q=O_{p^{\prime}}(G) \neq 1$. Let $H=Q N_{G}(P)$. If $H \neq G$, then $P \triangleleft H$ by induction. Hence

$$
O_{p^{\prime}, p}(H)=O_{p^{\prime}}(H) \times P \geqslant O_{p^{\prime}, p}(G) \underset{\neq}{>} Q
$$

because $G$ is $p$-solvable. This forces $O_{p}(G) \neq 1$, a contradiction. Therefore, $G=H$. But $G=O^{p^{\prime}}(G)$ by minimality, whence $G=Q P$. In particular, $G$ is $p$-nilpotent, and every $p$-block $B$ of $G$ contains only one modular character by Theorem 14.9 of [6]. Since $P$ is a T.I. Sylow $p$-subgroup, and $\chi(1)<|P|$ it follows from Theorem 14.8 of [6] that (the possibly reducible) $\chi$ contains an irreducible constituent $\mu$ belonging to a nonprincipal $p$-block $B$ with defect group $\delta(B)={ }_{G} P$, because $\chi$ is faithful. As $G$ is $p$-nilpotent, by Theorem 2.1 of $[1$, p. 419], we also may assume that $\mu$ remains irreducible under restriction modulo $\pi$. Let $\bar{\mu}$ be a module over $F$ affording $\mu$ modulo $\pi$.

Let $b$ be the block of $U=N_{G}(P)$ associated with $B$ by the Brauer correspondence. Since $\mu(1)^{2}<|P|$, Lemma 1.1 asserts that $\left.\mu\right|_{U}$ is irreducible. Because $P$ is a T.I. set, Green's correspondence theorem implies that $\left.\bar{\mu}\right|_{U}$ is an indecomposable module in $b$. Notice that $b$ contains only one modular irreducible character, so that all composition factors of $\left.\bar{\mu}\right|_{U}$ are isomorphic. In particular, if $\left.\bar{\mu}\right|_{U}$ is not irreducible, it has a nonzero nilpotent $F U$-endomorphism $\tau$, namely any nonzero map from the head to the socle of $\left.\bar{\mu}\right|_{U}$. As $P$ is a T.I. set, Corollary 5.8 of [9, p. 122], implies that $\tau$ is a projective endomorphism of $\left.\bar{\mu}\right|_{U}$. Therefore, $\mu(1)^{2}>|P|$ by Corollary 6.11 of $\left[9\right.$, p. 128], a contradiction. We now know that $\left.\bar{\mu}\right|_{U}$ is an irreducible $F U$-module of $b$ and that $P$ acts trivially on $\left.\bar{\mu}\right|_{U}$. Let $A=\operatorname{ker} \bar{\mu}$ in $G$. Now $A \neq G$ since $B$ is not the principal block. We have

$$
P \leqslant \operatorname{ker} \bar{\mu}=A<G .
$$

Therefore, $Q \nless A$ as $G=Q P$.

By Lemma $1.2 P$ is a T.I. Sylow $p$-subgroup of $A$, and $\chi_{A}$ is a faithful complex 
character of $A$ with $\chi_{A}(1)^{2}<|P|$. So $P \triangleleft A$ by induction, which implies $P \triangleleft G$. This contradiction completes the proof.

The center of the group $G$ is denoted by $Z(G)$.

Proposition 1.4. Let $G$ be a minimal counterexample to Leonard's conjecture. Let $P$ be a T.I. Sylow p-subgroup of $G$, and let $\chi$ be a faithful complex character of degree $\chi(1) \leqslant \sqrt{|P|}-1$. Then:

(a) $Z(G)=O_{p^{\prime}}(G)<O^{p}(G)=H, G=O^{p^{\prime}}(G)$.

(b) $H / Z(G)$ is a nonabelian simple group with a T.I. Sylow p-subgroup.

(c) $\chi$ may be assumed to be irreducible.

Proof. As $G$ is a minimal counterexample, $G=O^{p^{\prime}}(G)$. Let $H=O^{p}(G)$, and let $H / N \neq 1$ be a chief factor of $G$.

Suppose that $H / N$ is a $p^{\prime}$-group. By Proposition $1.3 G$ is not $p$-solvable. Thus $P_{0}=P \cap N \neq 1$, and $L=N_{G}\left(P_{0}\right)<G$. Since $P \cap N \triangleleft P, P \leqslant L$. So by induction $P \triangleleft L$. As $G=O^{p^{\prime}}(G)$, and as $G=N L$ by the Frattini argument, we obtain $G=N P$, and so $N=H$, a contradiction.

Therefore $H / N$ is a direct product of isomorphic nonabelian simple groups $A$ with $p|| A \mid$. Hence $N N_{G}(P)<G$, which implies $P \triangleleft N N_{G}(P)$ by induction. Since $P$ is a T.I. set in $G$, we now get $O_{p}(N)=N \cap P=1$. So $N$ is a $p^{\prime}$-group commuting with $P$. Hence

$$
C_{G}(N) \geqslant\left\langle P^{g} \mid g \in G\right\rangle=O^{p^{\prime}}(G)=G,
$$

and so $Z(G)=N \leqslant O_{p^{\prime}}(G)$.

As $(p,|N|)=1$, Lemma 1.2 asserts that $H / N$ has a T.I. Sylow $p$-subgroup. Thus by Lemma $1.2(\mathrm{c}) H / N$ is simple. Since $O_{p^{\prime}}(G) \subset O^{p}(G)=H$, it follows that $N=O_{p^{\prime}}(G)$.

Finally, we may replace $\chi$ by an irreducible constituent, which does not have $H$ in its kernel. This completes the proof.

2. Simple groups with a noncyclic T.I. Sylow $p$-subgroup. In this section we list the simple groups with a noncyclic T.I. Sylow $p$-subgroup. In [13] Suzuki classified the simple groups with such a Sylow 2-subgroup. For odd primes $p$ our subsidiary result follows from Gorenstein and Lyons' classification [4] of the finite groups $G$ with $O_{p^{\prime}}(G)=1, p$-rank $m_{p}(G)>1$, and containing a strongly $p$-embedded subgroup.

Here $m_{p}(G)$ denotes the maximum rank of an elementary abelian subgroup of a Sylow $p$-subgroup $P$ of the finite group $G$.

Definition [3]. Let $P$ be a Sylow $p$-subgroup of the finite group $G$, and let $k$ be a positive integer. The $k$-generated $p$-core of $G$ is $\Gamma_{P, k}(G)=\left\langle N_{G}(Q)\right| Q \leqslant P, m_{p}(Q)$ $\geqslant k\rangle$.

The proper subgroup $M$ of $G$ is called strongly p-embedded in $G$ if $\Gamma_{P, 1}(G) \leqslant M$.

REMARK 2.1. If the finite group $G$ contains a nonnormal T.I. Sylow $p$-subgroup $P$, then $M=N_{G}(P)$ is strongly $p$-embedded in $G$, as is easily seen.

A finite group $G$ is quasi-simple if $G=G^{\prime}$ and $G / Z(G)$ is simple. The layer $L(G)$ of $G$ is the product of all subnormal quasi-simple subgroups of $G$, where $L(G)=1$ if no such subnormal subgroup exists. The generalized Fitting subgroup of the finite group $G$ is defined as $F^{*}(G)=F(G) L(G)$, where $F(G)$ denotes the Fitting subgroup of $G$ (see [3, p. 44]). 
In view of the classification theorem of the finite simple groups we now can restate Theorems (24.1), (24.2), and (24.9) of Gorenstein and Lyons [4, pp. 307, 311, and 318 , respectively], as

Proposition 2.2. Let $p$ be an odd prime, $M$ a strongly p-embedded subgroup of the finite group $G$ with $O_{p^{\prime}}(G)=1$ and $m_{p}(G)>1$. Let $V=O^{p^{\prime}}(G)$ and let $P$ be a Sylow p-subgroup of $M$. Then $F^{*}(G)=L(V)$ is simple and one of the following holds.

(1) $V \cong \operatorname{PSL}_{2}\left(p^{n}\right)$ or $\operatorname{PSU}_{3}\left(p^{n}\right)$, and $M=N_{G}(P)$.

(2) $V \cong \mathfrak{U}_{2 p}$ and $F^{*}(M) \cong \mathfrak{U}_{p} \times \mathfrak{U}_{p}$.

(3) $p=3, V \cong{ }^{2} G_{2}\left(3^{2 m+1}\right)$, and $M=N_{G}(P)$, where $m \geqslant 0$.

(4) $p=3, V \cong M_{11}$ or $\operatorname{PSL}_{3}(4)$, and $M=N_{G}(P)$.

(5) $p=5, V \cong M(22)$, and $V \cap M \cong \operatorname{Aut}\left(D_{4}(2)\right)$.

(6) $p=5, V \cong{ }^{2} F_{4}(2)^{\prime}$, Aut $\left({ }^{2} B_{2}\left(2^{5}\right)\right)$ or $M c$, and $M=N_{G}(P)$.

(7) $p=11, V \cong J_{4}$, and $M=N_{G}(P)$.

Proof. By hypothesis, $\Gamma_{P, 1}(G) \leqslant M \neq G$ and $P \leqslant V \cap M$. Thus $O_{p}(G)=1=$ $F(G)$, because otherwise $G=N_{G}\left(O_{p}(G)\right) \leqslant \Gamma_{P, 1}(G) \leqslant M \neq G$, a contradiction.

Let $K$ be a normal subgroup of $G$. As $O_{p^{\prime}}(G)=1, P_{0}=P \cap K \neq 1$. The Frattini argument asserts that $G=N_{G}\left(P_{0}\right) K$. Hence $K \nless \Gamma_{P, 1}(G)$. It follows that every quasi-simple subnormal subgroup $L$ of $G$ is simple and $L \neq \Gamma_{P_{1}, 1}(L)$, where $P_{1} \in \operatorname{Syl}_{p}(L)$.

Thus $F^{*}(G)=L(G)=L(V)$ is a direct product of simple groups $E_{i}, 1 \leqslant i \leqslant k$, each of which contains a strongly $p$-embedded subgroup.

Let $E \in\left\{E_{i} \mid 1 \leqslant i \leqslant k\right\}, P^{*}=P \cap L(V)$, and $X=E P^{*}$. Then $O_{p^{\prime}}(X)=1=$ $O_{p}(X)$ and $\Gamma_{P^{*}, 1}(X) \neq X$, because $P^{*} \subset \Gamma_{P, 1}(G)$, but $E \nless \Gamma_{P, 1}(G)$. Applying now Theorem (24.9)(4) of Gorenstein and Lyons [4, p. 318], we obtain that $\Omega_{1}\left(P^{*}\right) \leqslant E$ or $E \in\left\{G_{2}(3)^{\prime},{ }^{2} B_{2}\left(2^{5}\right)\right\}$. Since $P^{*} \in \operatorname{Syl}_{p}(L(V))$ it follows that $P^{*} \leqslant E$. As $O_{p^{\prime}}(L(V))=1$ we get $F^{*}(G)=L(G)=L(V)=E$. Hence $F^{*}(G)$ is simple. Now Theorems (24.1) and (24.2) of Gorenstein and Lyons [4, pp. 307, 311] complete the proof.

Combining this result with Suzuki's theorem [13] we obtain

Proposition 2.3. Let $G$ be a nonabelian simple group with a noncyclic T.I. Sylow p-subgroup $P$. Then $G$ is isomorphic to one of the following groups.

(a) $\operatorname{PSL}_{2}(q)$ or $\operatorname{PSU}_{3}(q)$, where $q=p^{n}$ and $n \geqslant 2$ or $n \geqslant 1$, respectively.

(b) $p=2$ and $G \cong{ }^{2} B_{2}\left(2^{2 m+1}\right)$.

(c) $p=3$ and $G \cong{ }^{2} G_{2}\left(3^{2 m+1}\right)$, where $m \geqslant 1$.

(d) $p=3$ and $G \cong \mathrm{PSL}_{3}(4)$ or $M_{11}$.

(e) $p=5$ and $G \cong{ }^{2} F_{4}(2)^{\prime}$ or $M c$.

(f) $p=11$ and $G \cong J_{4}$.

Proof. If $p=2$, then (a) and (b) follow from Theorem 1 of [13].

Let $p$ be odd. By Remark $2.1 G$ can only be one of the simple $L(V)$ occurring in the list of Proposition 2.2. Since $\mathfrak{A}_{p} \times \mathfrak{A}_{p}$ is a subgroup of $\mathfrak{A}_{2 p}$, Lemma 1.2 asserts that $G \equiv \mathfrak{A}_{2 p}$. A group $H$ with a T.I. Sylow $p$-subgroup has only $p$-blocks of defect zero and of highest defect. By the character table system CAS [11] $M(22)$ has a 5-block of defect one. Thus $G \not M(22)$. Since $\operatorname{Aut}\left({ }^{2} B_{2}\left(2^{5}\right)\right)$ is not simple, $G \not$ $\operatorname{Aut}\left({ }^{2} B_{2}\left(2^{5}\right)\right)$. 
Now $\operatorname{PSL}_{2}\left(p^{n}\right)$ and $\operatorname{PSU}_{3}\left(p^{n}\right)$ have T.I. Sylow $p$-subgroups (see [5, pp. 191, 242]). By Ward [14] ${ }^{2} G_{2}\left(3^{2 m+1}\right)$ has a T.I. Sylow 3-subgroup. As can be seen from the character table of $\mathrm{PSL}_{3}(4)$ the Sylow 3-subgroup $P$ equals $C_{G}(x)$ for every $1 \neq x \in P$. Hence $P$ is a T.I. set.

It is well known and easy to check that the Sylow 3-subgroups of $M_{11}$ and the Sylow 5-subgroups of the Tits group ${ }^{2} F_{4}(2)^{\prime}$ and the McLaughlin group $M c$ are T.I. By Propositions 22 and 26 of Janko [7] the Sylow 11-subgroups of $J_{4}$ are T.I. This completes the proof.

3. Proof of the main result. In this section, Leonard's conjecture is proved by means of the results mentioned above.

Let $G$ be a finite group with a Sylow $p$-subgroup $P$. If $C_{G}(P)=C_{G}(x)$ for every $1 \neq x \in P$, then $P$ is called weakly self-centralizing. The following lemma is well known.

Lemma 3.1. Let $G$ be a finite group with a cyclic Sylow p-subgroup $P$. Then $P$ is a $T . I$. set if and only if $P$ is weakly self-centralizing.

THEOREM 3.2. Let $G$ be a finite group with a T.I. Sylow p-subgroup P. If $G$ has a faithful complex character $\chi$ with degree $\chi(1) \leqslant \sqrt{|P|}-1$, then $P$ is a normal subgroup of $G$.

Proof. If $P$ is cyclic, then $P$ is weakly self-centralizing. As $\sqrt{|P|}-1<\frac{1}{2}(|P|-1)$ for every prime $p>0$, it follows from Sibley's theorem [12] that $P \triangleleft G$.

Now let $G$ be a counterexample of minimal order. Then $P$ is not cyclic, $G=O^{p^{\prime}}(G)$, and by Proposition $1.4 Z=Z(G)=O_{p^{\prime}}(G)<O^{p}(G)=H$. Furthermore, $H / Z$ is a nonabelian simple group with a T.I. Sylow $p$-subgroup, and we may assume that $\chi$ is irreducible. We also can assume that $G$ does not have a proper abelian direct factor.

Suppose that $p$ is odd. Then $m_{p}(G)>1$, because $P$ is not cyclic. By Remark 2.1 $N_{G}(P)$ is strongly $p$-embedded in $G$. Therefore it follows from Propositions 2.2 and $p=3$ and $G \cong{ }^{2} G_{2}(3)$ or 2.3 that $H=O^{p}(G)=G$ except when $p=3$ and $G \cong{ }^{2} G_{2}(3)$ or $p=5$ and $G / Z \cong \operatorname{Aut}\left({ }^{2} B_{2}\left(2^{5}\right)\right)$. Now remembering that $p+|Z|$ and using Gorenstein's table [3, Table 4.1, p. 302] of the Schur multipliers of the finite simple groups, the structure of $G$ can be described as in the following table.

\begin{tabular}{l|l|l} 
prime $p$ & \multicolumn{1}{|c|}{$G / Z$} & \multicolumn{1}{c}{$Z$} \\
\hline$p \mid q$ & $\operatorname{PSL}_{2}(q)$ or $\operatorname{PSU}_{3}(q)$ & $|Z| \leqslant 2$ or $|Z| \leqslant 3$ \\
\hline$p=3$ & ${ }^{2} G_{2}\left(3^{2 m+1}\right)$ & $Z=1$ \\
\hline$p=3$ & $\operatorname{PSL}_{3}(4)$ or $M_{11}$ & $|Z| \leqslant 4$ or $Z=1$ \\
\hline$p=5$ & ${ }^{2} F_{4}(2)^{\prime}$ & $Z=1$ \\
\hline$p=5$ & $\operatorname{Aut}\left({ }^{2} B_{2}\left(2^{5}\right)\right)$ & $Z=1$ \\
\hline$p=5$ & $M c$ & $|Z| \leqslant 3$ \\
\hline$p=11$ & $J_{4}$ & $Z=1$ \\
\hline
\end{tabular}


Applying the theorem of Landazuri and Seitz [8] on the minimal degrees of the nontrivial complex projective representations $\pi$ of $\operatorname{PSL}_{2}(q), \operatorname{PSU}_{3}(q)$, or ${ }^{2} G_{2}\left(3^{2 m+1}\right)$ we see that $\pi(1) \geqslant \frac{1}{2}(q-1), \pi(1) \geqslant q(q-1)$, and $\pi(1) \geqslant 3^{2 m+1}\left(3^{2 m+1}-1\right)$, respectively. In any case $\pi(1)>\sqrt{|P|}-1$, a contradiction. If $p=3$ and $G / Z \in$ $\left\{\operatorname{PSL}_{3}(4), M_{11}\right\}$, then $|P|=9$. But another contradiction is obtained since the nontrivial irreducible projective characters of these simple groups have minimal degrees

$$
\chi(1)= \begin{cases}4, & \text { if } G / Z=\operatorname{PSL}_{3}(4), \\ 10, & \text { if } G=M_{11} .\end{cases}
$$

If $p=5$ and $G={ }^{2} F_{4}(2)^{\prime}$ then every nontrivial irreducible character $\chi$ of $G$ has degree $\chi(1) \geqslant 26$. However, $|P|=25$, a contradiction.

If $p=5$ and $G=\operatorname{Aut}\left({ }^{2} B_{2}\left(2^{5}\right)\right)$, then every faithful irreducible character $\chi$ of $G$ has degree $\chi(1) \geqslant \pi(1)$, where $\pi$ is a nontrivial irreducible character of the Suzuki group ${ }^{2} B_{2}(q), q=2^{5}$, of minimal degree. Now by Landazuri and Seitz [8, p. 419], $\pi(1)=4 \cdot 31=124$. Since $|P|=125$, we obtain $\chi(1)>\sqrt{|P|}-1$, a contradiction.

If $p=5$ and $G / Z=M c$, then every irreducible nontrivial character $\chi$ of $G$ has degree $\chi(1) \geqslant 22$ by the character table of $M c$ (see [11]). As $|P|=125, G$ cannot be a minimal counterexample. If $G / Z \cong M c$ and $|Z|=3$, we again use the character table of $G$ (see [11]) and find that the nontrivial projective irreducible character $\chi$ of minimal degree has degree $\chi(1)=126 \geqslant \sqrt{125}-1$, another contradiction.

If $p=11$ and $G=J_{4}$, then $\chi(1) \geqslant 1333$ by [11]. Since $|P|=11^{3}, \chi(1)>\sqrt{|P|}-1$, which is impossible by hypothesis.

Therefore $p=2$. Hence by Theorem 2 of Suzuki [13] and Proposition 1.4 we get $G / Z \in\left\{\operatorname{PSL}_{2}(q), \mathrm{PSU}_{3}(q),{ }^{2} B_{2}(q)\right\}$, where $q$ is a power of 2 . Using the theorem of Landazuri and Seitz [8] as above, we obtain our final contradiction. This completes the proof.

REMARK 3.3. It is not possible to replace the bound $\sqrt{|P|}-1$ by the bound $\frac{1}{2}(|P|-1)$ of Sibley's theorem [12]. Let $G=M c, p=5$, and $\chi$ be the irreducible character of $G$ with degree $\chi(1)=22$. The Sylow 5-subgroup $P$ of $G$ is T.I. and has order $|P|=5^{3}=125$. Hence $\chi(1)=22<62=\frac{1}{2}(|P|-1)$. However, $P$ is not normal. In particular, Sibley's condition that $P$ be weakly self-centralizing cannot be weakened to $P$ being a T.I. set.

Acknowledgment. The authors are grateful to M. Broué and L. Puig who suggested applying Gorenstein and Lyons' work [4] in the proof of Proposition 2.3. Our original proof of this result was based on a case-by-case analysis of the structure of the centralizers of the semisimple and unipotent elements of the finite simple groups of Lie type. We also thank H. Blau and the referee for several helpful remarks.

The first two authors gratefully acknowledge financial support by the Deutsche Forschungsgemeinschaft, Bonn, West Germany, enabling each of them to visit Essen University within the periods April-August, 1983, and May-June, 1984, respectively. 


\section{REFERENCES}

1. W. Feit, The representation theory of finite groups, North-Holland, Amsterdam, 1982. MR 83g 20001

2. D. Gorenstein, Finite groups, Harper \& Row, New York, 1968. MR 38229

3. __ Finite simple groups, Plenum Press, New York, 1982. MR 84j 20002

4. D. Gorenstein and R. Lyons, On finite groups of characteristic 2-type, Mem. Amer. Math. Soc. No. 276 (1983). MR 82h 20021

5. B. Huppert, Endliche Gruppen. I, Springer, Heidelberg, 1967. MR 37302

6. B. Huppert and N. Blackburn, Finite groups. II, Springer, Heidelberg, 1982. MR 84i 20001a

7. Z. Janko, A new finite simple group of order 86775571046077562880 which possesses $M_{24}$ and the full cover of $M_{22}$ as subgroups, J. Algebra 42 (1976), 564-596. MR 555734

8. V. Landazuri and G. M. Seitz, On the minimal degrees of projective representations of the finite Chevalley groups, J. Algebra 32 (1974), 418-443. MR 5013299

9. P. Landrock, Finite group algebras and their modules, Cambridge Univ. Press, Cambridge, 1983.

10. H. S. Leonard, On finite groups whose p-Sylow subgroup is a T.I. set, Proc. Amer. Math. Soc. 19 (1968), 667-670. MR 374168.

11. J. Neubüser, H. Pahlings, and W. Plesken, CAS; Design and use of a system for the handling of characters of finite groups, Proc. Conf. on Computational Group Theory, Academic Press, London, 1984, pp. 195-247.

12. D. A. Sibley, Finite linear groups with a strongly self-centralizing Sylow subgroup. II, J. Algebra 36 (1975), 319-332. MR 523305.

13. M. Suzuki, Finite groups of even order in which Sylow 2-groups are independent, Ann. of Math. 80 (1964), 58-77. MR 29145

14. H. N. Ward, On Ree's series of simple groups, Trans. Amer. Math. Soc. 121 (1966), 62-89. MR 33 5752

Department of Mathematics, University of Minnesota, Minneapolis, Minnesota 55455

Department of Mathematics, University of Aarhus, 8000 Aarhus C, Denmark

Department of Mathematics, University of Essen, 4300 Essen, West Germany 